\title{
Towards optimal post-fault self-healing in future smart distribution grids
}

\author{
Florin Capitanescu, Ilya Bilibin and Juergen Sachau \\ Interdisciplinary Centre for Security, Reliability and Trust \\ University of Luxembourg | Faculty of Science, Technology and Communication 4 \\ Phone: +352466644 5721; fax: +3524666445669. \\ Emails: Florin.Capitanescu@uni.lu, Ilya.Bilibin@uni.lu, Juergen.Sachau@uni.lu.
}

\begin{abstract}
This paper deals with a key functionality of the future distribution smart grids namely the automatic post-fault network self-healing. To this end we propose an optimization approach that aims finding the post-fault grid topology that minimizes the amount of unsupplied energy to consumers. The approach extends existing optimization models, developed in the context of losses minimization, for our particular application and proposes ways to overcome their limitations. We prove the interest of the approach on a 33-bus benchmark distribution system as well as on a 61-bus real-life distribution grid.
\end{abstract}

\section{Key words}

Smart grids, service restoration, optimal power flow, grid self-healing, network reconfiguration.

\section{Introduction}

Due to economical and protection selectivity reasons distribution systems are generally operated radially and each feeder is equipped with a single breaker; hence whenever there is a fault on a feeder all consumers fed from it remain temporarily unsupplied while renewable distributed generators connected to the feeder are temporarily lost.

Restoring the service (or supply) in an optimal way after fault isolation [1] is an important mean for improving distribution grid reliability, and is expected to become a key feature of the envisioned future smart distribution grids, under the ambitious appellation of automatic postfault distribution grid self-healing [2].

The problem of post-fault service restoration in current distribution systems consists in its general form in finding the optimal sequence of switching actions along with the optimal crew dispatch (i.e. in terms of number of crews and their timetable) that minimize the amount of post-fault unsupplied energy and avoid violating operational constraints (e.g. thermal and voltage) [1]. This general problem is very complex due to several reasons (i.e. nonlinearity, non-convexity, presence of binary variables, the optimal number of switching actions is not known beforehand, size which is proportional with the number of switching actions, etc.) and requires short computational effort which precludes the use of available mathematical programming solvers. In order to break-down its complexity and meet stringent computational time requirements, this optimization problem is typically decomposed into three sequential steps [3] at the expense of obtaining sub-optimal solutions:

1) finding the optimal post-fault final network configuration;

2) finding the optimal sequence of switching actions leading to the final network configuration without (severely) violating operational constraints and assuming that a feasible sequence exists for the optimal final grid topology;

3) dispatching optimally the crews to implement the optimal switching sequence.

The problem decomposition is also justified by several practical reasons:

- the distribution system operators (DSOs) have general very good field expertise regarding steps 2 and 3 ,

- the optimal sequence of control actions can be straightforwardly determined by an enumerative approach (which explores by a classical power flow program grid configurations for switching actions, possibly using parallel computations) as long as the number of switching actions is relatively small,

- obviously the larger the number of crews, the faster the restoration process, and hence the better the objective.

These observations lead to emphasize even more the role of the first step of the decomposed service restoration procedure. Accordingly, most researches on service restoration focus on the first step of the decomposed approach by: composite heuristics [4-10], evolutionary algorithms [11-15], and expert systems [16] among others, for a comprehensive literature survey up to 2010 about the entire service restoration topic the reader is referred to [1].

In their transition towards smarter distribution systems [2], it is expected that distribution systems will be 
equipped at strategic locations with an increasingly significant number of remotely controlled switches or reclosers that will greatly facilitate the fast and possibly automatic grid control.

In this paper we focus on the first step of the decomposed service restoration procedure adopting an approach that may fit both purposes: a fully automatic grid self-healing in future envisioned smart distribution grids and a combination of manually and remotely controlled switching in current distribution networks. This problem can be cast in its general form as a mixed integer nonlinear programming (MINLP) problem. However, solvers of this class of optimization problems are not able to comply with the stringent time requirements of the online operation [17]. Fortunately, as most distribution systems are operated radially, there is possible to reformulate the MINLP problem as a much more tractable equivalent mixed integer quadratically constrained program (MIQCP), as demonstrated in [18, 19] for the power losses minimization by means of network reconfiguration. The main contribution of this paper is to adapt and further develop the MIQCP model in $[18,19]$ to the problem of service restoration.

The remaining of the paper is organized as follows. Section 2 presents the optimization model of the proposed approach. Section 3 provides numerical results with the approach and section 4 concludes.

\section{Proposed approach}

The optimization model followed in this paper adapts the models proposed in [18] and [19] for the problem of service restoration. These models rely in turn on the power flow model for radial distribution systems proposed in [20]. The model uses a branch quadruple model as shown in Figure 1, in which the notations are selfexplanatory.

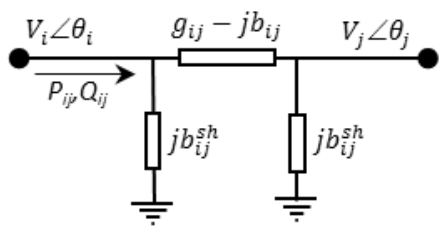

Fig. 1. Branch model.

\section{A. Alternative power flow equations for radial distribution systems}

The model proposed in [20] consists, for a grid with $n+1$ buses, in replacing the $2 n$ conventional nonlinear power flow equations with a set of $3 n$ equations ( $2 n$ linear and $n$ quadratic). This can be done by replacing the conventional complex voltage unknowns with two variables per branch $\left(W_{i j}\right.$ and $\left.T_{i j}\right)$ and one per bus $\left(U_{i}\right)$ as follows:

$$
\begin{gathered}
U_{i}=V_{i}^{2}, \\
W_{i j}=V_{i} V_{j} \cos \left(\theta_{i}-\theta_{j}\right), \\
T_{i j}=V_{i} V_{j} \sin \left(\theta_{i}-\theta_{j}\right) .
\end{gathered}
$$

where $V_{i}$ and $\theta_{i}$ are the magnitude and angle of the complex voltage at bus $i$, and $W_{i j}=W_{j i}$ and $T_{i j}=-T_{i j}$.

The new variables are related among each other according to:

$$
U_{i} U_{j}=W_{i j}^{2}+T_{i j}^{2}
$$

Thanks to these new variables the branch active and reactive power flow take on linear expressions:

$$
\begin{gathered}
P_{i j}=g_{i j} U_{i}-g_{i j} W_{i j}+b_{i j} T_{i j}, \\
Q_{i j}=\left(b_{i j}-b_{i j}^{s h}\right) U_{i}-b_{i j} W_{i j}-g_{i j} T_{i j} .
\end{gathered}
$$

Therefore the set of power flow equations proposed in [20] is made of $n$ the quadratic constraints (4) together with the $2 n$ linear equations:

$$
\begin{gathered}
P_{g i}-P_{c i}=\sum_{j \in B_{i}} P_{i j}= \\
=\sum_{j \in B_{i}}\left(g_{i j} U_{i}-g_{i j} W_{i j}+b_{i j} T_{i j}\right), \\
Q_{g i}-Q_{c i}=\sum_{j \in B_{i}} Q_{i j}= \\
=\sum_{j \in B_{i}}\left(\left(b_{i j}-b_{i j}^{s h}\right) U_{i}-b_{i j} W_{i j}-g_{i j} T_{i j}\right) .
\end{gathered}
$$

where $B_{i}$ is the set of branches connected to bus $i, P_{g i}$ and $P_{c i}$ (respectively $Q_{g i}$ and $Q_{c i}$ ) are the active (respectively reactive) power generated and consumed at bus $i$.

The voltages magnitude and angle can be retrieved straightforwardly from the solution of these new equations by exploring the graph tree downward from the substation slack bus [20]:

$$
\begin{gathered}
V_{i}=\sqrt{U_{i}} \\
\theta_{i}=\theta_{j}+\arctan \left(T_{i j} / W_{i j}\right)
\end{gathered}
$$

\section{B. Modeling the branch switching operation}

In order to represent the network switching operation and consequently whether a branch is connected or disconnected we use the set of constraints proposed in [19]. The latter introduce two new variables per branch ( $U_{i}^{l}$ and $U_{j}^{l}$ ) and one binary variable $\alpha_{l}$ to describe the switch status (on or off) of every branch:

$$
\begin{aligned}
0 & \leq U_{i}^{l} \leq \alpha_{l} V_{i \text { max }}^{2}, \\
0 \leq U_{j}^{l} & \leq \alpha_{l} V_{j \text { max }}^{2}, \\
0 \leq U_{i}-U_{i}^{l} & \leq\left(1-\alpha_{l}\right) V_{i \text { max }}^{2}, \\
0 \leq U_{j}-U_{j}^{l} & \leq\left(1-\alpha_{l}\right) V_{j \text { max }}^{2}
\end{aligned}
$$

and rewrite the active and reactive power flows (5) and (6) as well as the quadratic constraints (4) in terms of new variables:

$$
\begin{gathered}
P_{i j}=g_{i j} U_{i}^{l}-g_{i j} W_{i j}+b_{i j} T_{i j}, \\
Q_{i j}=\left(b_{i j}-b_{i j}^{s h}\right) U_{i}^{l}-b_{i j} W_{i j}-g_{i j} T_{i j}, \\
U_{i}^{l} U_{j}^{l}=W_{i j}^{2}+T_{i j}^{2} .
\end{gathered}
$$


Observe that if then $\alpha_{l}=0$ and from (17) one obtains $W_{i j}=T_{i j}=0$ and finally from (15) and (16) one obtains $P_{i j}=Q_{i j}=0 \quad$ (likewise $P_{j i}=Q_{j i}=0$ ), which models properly the desired effect of branch disconnection. On the other hand if $\alpha_{l}=1$ then from $(13,14)$ one gets $U_{i}=U_{i}^{l}$ and $U_{j}=U_{j}^{l}$ and the equations (15)-(17) recover the equations (4)-(6) of the connected branch.

\section{The model of the optimization problem}

Adapting and extending the models proposed in [18] and [19] for the problem of service restoration leads to the optimization problem described hereafter.

The goal of the optimization problem is to minimize the amount of unsupplied load. However, very often the optimal value of unsupplied load could be reached by various switching actions (e.g. especially in the case where all load can be safely supplied). From a practical point of view (e.g. crew dispatch, switch lifetime) it is desirable to produce a practical solution which minimizes the number of switching operations. This leads to consider the following composite objective in which the weight $w$ is chosen to a very small value so as to give full precedence to the minimization of unsupplied load:

$$
\min \sum_{c} p_{i} s_{i} P_{c i}+w\left|\alpha_{l}-\alpha_{l}^{0}\right|
$$

where $p_{i}$ 's account for load priorities, $s_{i}$ is a binary variable modeling the fact that, as opposite to the transmission system, in distribution systems one cannot fed partially the load behind a MV/LV transformer, $\alpha_{l}^{0}$ is the initial status of switches just after fault isolation, and the penalty term $w\left|\alpha_{l}-\alpha_{l}^{0}\right|$ intends to discourage solutions involving too many switching maneuvers.

The problem is subject to the following constraints:

1) linear bus active and reactive power balance equations:

$$
\begin{gathered}
P_{g i}-s_{i} P_{c i}= \\
=\sum_{j \in B_{i}} g_{i j} U_{i}^{l}-g_{i j} W_{i j}+b_{i j} T_{i j}, \\
Q_{g i}-s_{i} Q_{c i}= \\
=\sum_{j \in B_{i}}\left(b_{i j}-b_{i j}^{s h}\right) U_{i}^{l}-b_{i j} W_{i j}-g_{i j} T_{i j} ;
\end{gathered}
$$

2) quadratic constraints completing the power flow equations:

$$
U_{i}^{l} U_{j}^{l}=W_{i j}^{2}+T_{i j}^{2}
$$

3) active power bounds on the equivalent slack generators at the substation:

$$
P_{g i}^{\min } \leq P_{g i} \leq P_{g i}^{\max }
$$

4) linear branch current limits:

$$
\begin{gathered}
I_{i j}^{2}=\left(g_{i j}^{2}+b_{i j}^{2}\right)\left(U_{i}^{l}+U_{j}^{l}-2 W_{i j}\right)- \\
-2 b_{i j} b_{i j}^{s h} W_{i j}+2 g_{i j} b_{i j}^{s h} T_{i j}+ \\
+\left(\left(b_{i j}^{s h}\right)^{2}+2 b_{i j} b_{i j}^{s h}\right) U_{i}^{l} \leq\left(I_{i j}^{\max }\right)^{2} ;
\end{gathered}
$$

5) linear constraints modeling the branch switch status:

$$
\begin{aligned}
0 & \leq U_{i}^{l} \leq \alpha_{l} V_{i \text { max }}^{2} \\
0 & \leq U_{j}^{l} \leq \alpha_{l} V_{j \text { max }}^{2} \\
0 \leq U_{i}-U_{i}^{l} & \leq\left(1-\alpha_{l}\right) V_{i \text { max }}^{2}, \\
0 \leq U_{j}-U_{j}^{l} & \leq\left(1-\alpha_{l}\right) V_{j \text { max }}^{2} ;
\end{aligned}
$$

6) voltage magnitude limits:

$$
V_{i \min }^{2} \leq U_{i}
$$

limits $U_{i} \leq V_{i \text { max }}^{2}$ being ensured by (24)-(27);

7) necessary radiality constraints:

$$
\sum_{l} \alpha_{l}=n
$$

together with additional constraints ensuring radiality that will be discussed later on.

The control variables of the problem are the branch switches status $\alpha_{l}$, the load curtailment status $s_{i}$, and slack generators active power $P_{g i}$, other optimization variables being: $U_{i}, U_{j}, U_{i}^{l}, U_{j}^{l}$, and $W_{i j}, T_{i j}$.

D. Overcoming some issues with this model and practical implementation aspects

a. Modifying the model to enable the use of appropriate optimization solvers

The model (18)-(29) is a mixed integer quadratically constrained program (MIQCP). However, trying to solve this model in GAMS by appropriate MIQCP solvers (e.g. CPLEX, MOSEK, XPRESS, etc.) in order to take advantage of the highly linear nature of the problem is not possible because the matrix of quadratic constraints (17) is not positively semi-definite. Therefore, this MIQCP problem remains to be solved by classical MINLP solvers (e.g. SBB, DICOPT, etc.) which, although will have to deal with simpler linear-quadratic NLP sub-problems, one wants avoiding.

A possible way to circumvent the use of MINLP solvers is to express the quadratic constraints (17) by weaker rotating quadratic cone constraints as in [19]:

$$
U_{i}^{l} U_{j}^{l} \geq W_{i j}^{2}+T_{i j}^{2}
$$

and formulate the optimization problem is such a way that these constraints are binding at the optimum. However, as opposed to [19], where the nature of the minimum power losses objective function drives naturally all these 
constraints to be binding at the optimum, this does not hold true for our problem. In order to enforce these constraints to be binding at the optimum we use a penalty term in the objective representing the overall power generated by the equivalent generators at substations:

$$
\min \sum_{c} p_{i} s_{i} P_{c i}+w\left|\alpha_{l}-\alpha_{l}^{0}\right|+\sum_{g} P_{g i}
$$

The role of this penalty term is to enforce the power flow equations to recover their solution, keeping in mind that in a radial distribution system the operationally feasible power flow solution is unique [21]. After extensive simulations with this approach we noticed that this heuristic worked well in all cases enabling thereby the use of adequate MIQCP solvers.

\section{b. On the radiality constraints}

Note that the necessary radiality constraint (29) may be however insufficient to ensure radiality in the presence of zero-injection nodes [18]. To avoid adding a significant number of new integer variables and constraints to ensure radiality as in [18] we adopt a practical solution which consists in replacing each zero-injection bus with a very small reactive power load (of value slightly above the power flow convergence tolerance), change which practically does not affect the final result of the optimization.

Furthermore, a major problem with the necessary radiality constraint (29) is that the number of buses that can be fed is not known apriorily and hence the constraint ensuring radiality may not hold. If there is a feasible switching solution to reroute power to all buses downstream the fault then the radiality necessary constraint holds. Otherwise, if some loads cannot be fed (e.g. especially in case of multiple faults due to storm or floods some loads may remain unfed without overloading the available feeders), in order to overcome the apriorily unknown number of closed switches we replace the radiality constraint (29) with the following one which expresses the fact that the number of closed switches equals the number of fed loads:

$$
\sum_{l} \alpha_{l}=\sum_{i} s_{i}
$$

Finally, a minor issue with the radiality constraints is that, in case of existing distributed generators (DGs), depending on their active and reactive power control rules, the current set of radiality constraints may not be able to prevent optimal solutions where the grid is islanded; in some islands the DGs perfectly fed locally a set of loads. If the grid code does not allow islanded operation then additional constraints are needed to prevent this case. To his end we use the set of constraints (11)-(15) which were proposed in [19] as necessary and sufficient constraints to ensure grid radiality but which additionally prevents grid islanding.

\section{Numerical results}

\section{A. Simulation assumptions}

The MIQCP optimization model has been developed in GAMS version 23.9.3 [23] and uses the IBM ILOG CPLEX solver, version 12.4.0.1. All tests have been performed on a PC of 2.8-GHz and 4-Gb RAM.

For the sake of assessing the efficiency of the optimization engines we assume that for all test systems all switches are remotely controlled.

\section{B. Tests on the 33-bus distribution network}

We first illustrate the approach on a $12.66 \mathrm{kV}$ benchmark distribution grid [22] with the following characteristics: 33 buses, one substation, 37 lines, 32 sectionalizing switches, and 5 tie switches. Figure 2 shows the one-line diagram of this network.

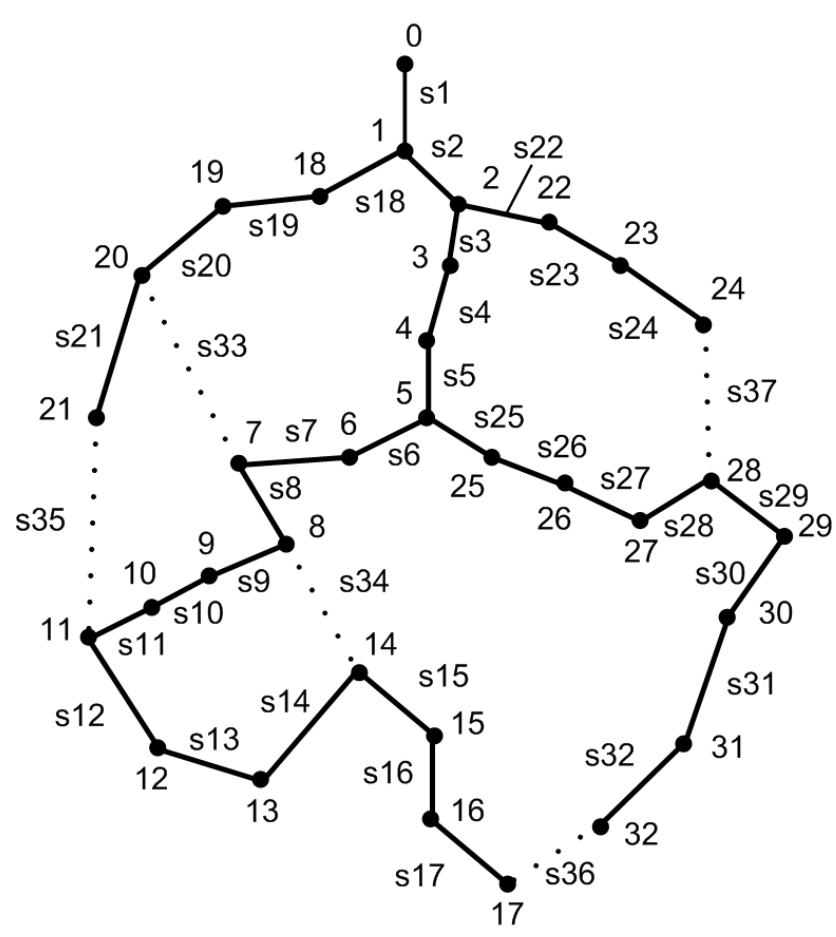

Fig. 2. Test network diagram.

The overall load in the base case is $3715 \mathrm{~kW}$ and 2300 $\mathrm{kVar}$ and most voltages lie between $0.90 \mathrm{pu}$ and $0.95 \mathrm{pu}$, the lowest voltage being of $0.904 \mathrm{pu}$.

We assume that a fault occured in the line linking nodes 2 and 3 and that the fault was isolated by opening the switch s3, which lets most loads of the grid unsupplied.

We solve the proposed optimization model for service restoration for four increasing values of the minimum voltage limit $V_{\min }$. We consider all 37 switches as control variables into the optimization.

Table 1 provides the results of these four simulations, where UL denotes the amount of unsupplied load while nbUL denotes the number of unsupplied loads. In cases A and $\mathrm{B}$ all consumers can be supplied while satisfying 
operating constraints, the number of switching actions being relatively small. In case $\mathrm{C}$ (respectively D) some loads are curtailed at buses 17 and 32 (respectively at buses: $3,15,16,17,31,32$ ) in order to satisfy the operating constraints, the number of switching actions being relatively larger. As expected the tighter the voltage limits the larger the amount of load unsupplied.

Given the large combinatorial space of the problem (i.e. 37 binary variables stemming from switches statuses and 32 binary variables stemming from load curtailment status) we conclude that the computational times are acceptable in all cases. In simple cases (e.g. A and B) where several feasible solutions exist the method is very fast. However, the cases that we artificially constrained very significantly (e.g. cases $\mathrm{C}$ and $\mathrm{D}$ ) the computational effort is larger in order to find a feasible final grid topology.

TABLE 1. SUMMARY OF SIMULATION RESULTS

\begin{tabular}{|c|c|c|c|c|c|c|}
\hline Case & $\begin{array}{c}V_{\min } \\
(\mathrm{pu})\end{array}$ & $\begin{array}{c}\mathrm{UL} \\
(\mathrm{kW})\end{array}$ & nbUL & $\begin{array}{c}\text { Open } \\
\text { switches }\end{array}$ & $\begin{array}{c}\text { Closed } \\
\text { switches }\end{array}$ & $\begin{array}{c}\text { Time } \\
(\mathrm{s})\end{array}$ \\
\hline $\mathrm{A}$ & 0.90 & 0 & 0 & $\mathrm{~s} 3, \mathrm{~s} 6$ & $\mathrm{~s} 33, \mathrm{~s} 37$ & 0.44 \\
$\mathrm{~B}$ & 0.93 & 0 & 0 & $\mathrm{~s} 3, \mathrm{~s} 9, \mathrm{~s} 27$ & $\begin{array}{c}\mathrm{s} 33, \mathrm{~s} 35, \\
\mathrm{~s} 37\end{array}$ & 1.37 \\
\hline $\mathrm{C}$ & 0.94 & 150 & 2 & $\begin{array}{c}\mathrm{s} 3, \mathrm{~s} 8, \\
\mathrm{~s} 17, \mathrm{~s} 26, \\
\mathrm{~s} 32\end{array}$ & $\begin{array}{c}\mathrm{s} 33, \mathrm{~s} 35, \\
\mathrm{~s} 37\end{array}$ & 12.6 \\
\hline & & & & $\begin{array}{c}\mathrm{s} 3, \mathrm{~s} 4, \mathrm{~s} 9, \\
\mathrm{~s} 15, \mathrm{~s} 16, \\
\mathrm{~s} 17, \mathrm{~s} 26 \\
\mathrm{~s} 31, \mathrm{~s} 32\end{array}$ & $\begin{array}{c}\mathrm{s} 33, \mathrm{~s} 35, \\
\mathrm{~s} 37\end{array}$ & 34.7 \\
\hline
\end{tabular}

\section{Tests on the 61-bus CREOS distribution network}

We now consider a real-life $20 \mathrm{kV}$ distribution network model provided by CREOS, the grid operator in Luxembourg. The model has the following characteristics: 61 buses, 2 feeders, 60 sectionalizing switches and 3 tie switches. We consider voltage limits of $0.95 \mathrm{pu}$ and 1.05 pu at all nodes.

We assume that a fault occurred in the line linking nodes 33 and 37, which leads to losing the whole load of the second feeder. As the operation constraints are not a concern nowadays in this grid, the first feeder can safely fed the whole load by closing the tie switch s62. The optimization process lasts 0.28 seconds.

In order to create a more difficult scenario, we decrease the thermal limit of the 3 tie switches to 0.1 MVA. The optimal solution leads in this case to closing the switches s62 and s63 and opening the sectionalizing switches s13 and s46. The $56.7 \mathrm{~kW}$ of load at bus 14 remain unsupplied. The optimization process lasts 0.69 seconds.

\section{Conclusions}

This paper has proposed a very computationally attractive MIQCP optimization approach for service restoration problem, which can be solved very efficiently by powerful state-of-the-art solvers such as CPLEX. Despite large combinatorial space the method is very fast for realistic operating conditions and acceptably fast for severely constrained operating conditions.

The approach can be used in both the nowadays distribution systems as well as in the future distribution smart grids as a fully automatic post-fault network selfhealing functionality.

A drawback of this optimization model is that the continuous relaxation of its binary variables, a feature that is exploited in many MINLP and MIQCP solvers, has no physical meaning as the model requires radial topology whereas the relaxed values of switches status may create meshed topologies which are not accounted for in the model. Accounting for meshed grid topologies involve adding nonlinear equations (10) that would lead to a tough MINLP problem destroying thereby the computational benefits of the MIQCP formulation.

Future work will be conducted in order to further extend this modes so as to compute the optimal sequence of control actions to steer optimally the system from the initial post-fault state to the optimal grid topology.

\section{Acknowledgments}

This work is conducted as part of the "Reliable and Efficient Distributed Electricity Generation in Smart Grids" (REDESG) project within the "CREOS-SnT Framework Program". The authors are supported by the Fonds National de la Recherche, Luxembourg (C11/SR/1278568).

IBM LOG CPLEX Optimization Studio has been used thanks to the IBM academic initiative.

\section{References}

[1] N. Perrier, B. Agard, P. Baptiste, J.-M. Frayert, A. Langevin, R. Pellerin, D. Riopel, M. Trapanier, “A survey of models and algorithms for emergency response logistics in electric distribution systems - Part II: contingency planning level", CIRRELT 2010, available online at: https://www.cirrelt.ca/documentstravail/cirrelt-2010-06.pdf.

[2] P. Sakis Meliopoulos, G. Cokkinides, R. Huang, E. Farantatos, S. Choi, Y. Lee, X. Yu, "Smart grid technologies for autonomous operation and control", IEEE Transactions on smart grid, vol. 2, no. 1, 2011, pp. 1-10.

[3] P.M.S. Carvalho, F.J.D. Carvalho, and L.A.F.M. Ferreira, "Dynamic Restoration of Large-Scale Distribution Network Contingencies: Crew Dispatch Assessment", IEEE PES Powertech conference, Lausanne, Switzerland, 2007.

[4] A.L. Morelato and A. Monticelli, "Heuristic search approach to distribution system restoration", IEEE Trans. on Power Delivery, Vol. 4, No. 4, 1989, pp. 2235-2241.

[5] J. Nahman and G. Strbac, "A new algorithm for service restoration in large scale urban networks" Elect. Power Energy Syst., vol. 29, 1994, pp. 181-192.

[6] K. Nan Miu, H.-D. Chiang, B. Yuan, and G. Darling, "Fast Service Restoration for Large-Scale Distribution Systems with Priority Customers and Constraints", IEEE Trans. on Power Systems, vol. 13, no. 3, November 1998, pp. 789-795.

[7] D. S. Popovic and R. M. Ciric, "A multi-objective algorithm for distribution networks restoration", IEEE 
Trans. on Power Delivery, vol. 14, no. 3, November 1999, pp. 1134-1141.

[8] S. P. Singh, G. S. Raju, G. K. Rao, and M. Afsari, "A heuristic method for feeder reconfiguration and service restoration in distribution networks", Int. Journal of Elect. Power Energy Syst., vol. 31, no. 7-8, pp. 309-314, 2009.

[9] M.R. Kleinberg, K. Miu, and H.-D. Chiang, "Improving Service Restoration of Power Distribution Systems Through Load Curtailment of In-Service Customers" IEEE Trans. on Power Systems, vol. 26, no. 3, 2011, pp. 1110-1117.

[10] J.S. Wu, K.L. Tomsovic, and S.S. Chen, "A Heuristic Search Approach to Feeder Switching Operations for Overload Faults, Unbalanced Flow and Maintenance", IEEE Trans. Power Delivery, Vol. 6, No. 4, 1991, pp. $1579-1585$

[11] A. Botea, J. Rintanen, and D. Banerjee, "Optimal Reconfiguration for Supply Restoration With Informed A* Search", IEEE Trans. on Smart Grids, vol 3. no. 2, pp. 583-593, 2012

[12] A. Mendes, N. Boland, P. Guiney, and C. Riveros, "Switch and Tap-Changer Reconfiguration of Distribution Networks Using Evolutionary Algorithms", IEEE Trans. on Power Systems, in press, 2012.

[13] Y. Kumar, B. Das, and J. Sharma, "Multiobjective, multiconstraint service restoration of electric power distribution system with priority customers", IEEE Trans. on Power Delivery, vol. 23, no. 1, 2008, pp. 261-270.

[14] V. J. Garcia and P. Franca, "Multiobjective service restoration in electric distribution networks using a local search based heuristic", Eur. J. Oper. Res., vol. 189, no. 3, 2008, pp. 649-705.

[15] S. Toune, H. Fudo, T. Genji, Y. Fukuyama, and Y. Nakanishi, "Comparative Study of Modern Heuristic Algorithms to Service Restoration in Distribution Systems",
IEEE Trans. Power Delivery, Vol. 17, No. 1, 2002, pp. $173-181$.

[16] C.C. Liu, S.J. Lee, and S.S. Venkata, "An Expert System Operational Aid for Restoration and Loss Reduction of Distribution System", IEEE Trans. Power Systems, Vol. 3, No. 2, 1988, pp. 619-626.

[17] L. Platbrood, S. Fliscounakis, F. Capitanescu, P. Panciatici, C. Merckx, and M. Ortega-Vazquez, "Deliverable D3.2: development of prototype software for system steady-state optimization of the European transmission system", PEGASE project, available on-line at http://www.fp7pegase.com, 2011.

[18] E. Romero Ramos, J. Riquelme Santos, and J. Reyes, "A simpler and exact mathematical model for the computation of the minimal power losses tree", Elect. Power Energy Syst., vol. 80, no. 5, pp. 562-571, 2010.

[19] R. Jabr, R. Singh, and B.C. Pal, "Minimum Loss Network Reconfiguration Using Mixed-Integer Convex Programming" IEEE Trans. on Power Systems, vol. 27, no. 2, 2012, pp. 1106-1115.

[20] A. Gomez Exposito and E. Romero Ramos, "Reliable load flow technique for radial distribution networks", IEEE Trans. on Power Systems, vol. 14, no. 3, August 1999, pp. 1063-1069.

[21] H.D. Chiang and M. Baran, "On the existence and uniqueness of load flow solution for radial distribution power networks ", IEEE Trans. on Circuits and Systems, vol. 37, no. 3, 1990, pp. 410-416.

[22] M.E. Baran and F.F. Wu, "Network Reconfiguration in Distribution Systems for Loss Reduction and Load Balancing", IEEE Trans. Power Delivery, Vol. 4, No. 2, 1989, pp. 1401-1497.

[23] B.A. McCarl, "GAMS User Guide", Version 23.8, 2012. Available online: www.gams.com. 